\title{
Instrumentos científicos en México
}

\section{Scientific instruments in Mexico}

\author{
Martin Jesper Larsson ${ }^{i}$ \\ i Postdoctorante, Centro de Investigación y de Estudios Avanzados/Instituto Politécnico Nacional. \\ Ciudad de México - México \\ orcid.org/0000-0002-8996-3772 \\ martin.larsson@outlook.es
}

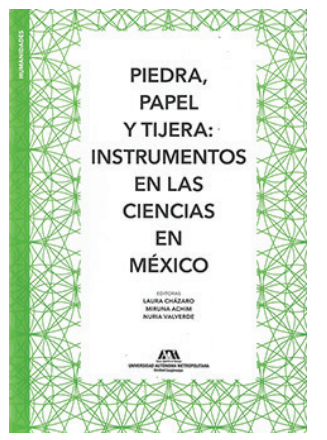

CHÁZARO, Laura; ACHIM, Miruna; VALVERDE, Nuria (Ed.). Piedra, papely tijera: instrumentos en las ciencias en México. Ciudad de México: Universidad Autónoma Metropolitana. 2018. $284 p$.

Piedra, papel y tijera: instrumentos en las ciencias en México (Cházaro, Achim, Valverde, 2018) es un conjunto de estudios que se centran en la historia de los diversos objetos que se han utilizado para construir, comunicar y aplicar conocimientos científicos. El libro se divide en 15 capítulos, que siguen tres ejes temáticos: "tecnologías de papel"; "prácticas y conocimientos médicos, materialidad in-corporada"; e "instrumentos, disponibilidad y objetividad en el México del siglo XX". Se indaga por los usos, los mercados, el trabajo manual y las habilidades de los objetos y artefactos de las ciencias, así como por la dimensión política de la creación, producción y circulación de éstos. Los objetos que aparecen en este libro muestran una flexibilidad y ambigüedad que dificulta una fijación espacial y temporal: los mismos objetos, al igual que las interpretaciones de ellos, dependen de sus relaciones con otros objetos, decisiones políticas, y de las personas que interactúan con ellos. En ese sentido, los objetos que se discuten se constituyen por un continuo devenir que es difícil de definir de antemano.

Un aspecto importante del libro es su énfasis en la interacción entre países productores y consumidores de los instrumentos que se discuten, visto desde la posición política de México. A través de esta mirada, lo político ocupa un lugar más central en las discusiones sobre instrumentos que lo común. Es el caso del estudio de la aparición de los primeros laboratorios, de Laura Cházaro, que subraya tanto las relaciones internacionales como la importancia política de la ciencia como una imagen útil para el gobierno federal. El estudio de Joel Vargas Domínguez es otro ejemplo que resalta la íntima conexión entre los estándares internacionales de calorías (establecidos en los EEUU) y las ideas de progreso en México. La relación entre estándares internacionales y usos locales es algo que también acentúa Natalia Coloballes en su estudio de la medición de la contaminación del aire en 
la Ciudad de México. En su capítulo, muestra cómo se desarrollaron instrumentos en México que funcionaban para distinguir entre diferentes ingredientes del polvo que había en el aire, pero que, con el tiempo, quedaron desplazados por instrumentos que correspondían a los estándares internacionales - no por su mayor capacidad de medición, sino por cuestiones políticas.

Como señala una de las editoras, Miruna Achim, el libro se inspira en el llamado "giro material", un giro que ha sido fundamental para el interés en instrumentos científicos, pero también para otros temas que se discuten, tales como los estándares y la infraestructura. ${ }^{1}$ Al mismo tiempo, el libro contiene contribuciones que claramente se inspiran en una materialidad de una tradición marxista. Si la idea de las editoras, como sugiere Laura Cházaro, era que el título subrayara el poder y la existencia frágil de los objetos que se ven reflejados en el juego infantil al cual hace alusión - donde las tijeras tienen el poder de cortar el papel, al mismo tiempo que pueden ser destruidas por la piedra - el título también se puede leer como una referencia a la tensión entre el giro material y el materialismo marxista.

Si esta tensión no es algo que se subraya en los textos del libro, resulta evidente al comparar los textos de Luis Sánchez y de Miruna Achim. Mientras Sánchez discute el papel que jugaron los instrumentos para la lucha de clases en la industria petrolera mexicana, el capítulo de Miruna Achim claramente destaca las particularidades materiales del papel como material, que se han vuelto invisibles en un entendimiento de los documentos como un medio entre actores. En este encuentro entre una mirada enfocada en una economía política, a menudo demasiado centrada en las relaciones humanas inmersas en estructuras de poder, y otra, que muchas veces ha sacrificado las relaciones de poder para hablar de las múltiples conexiones entre lo que comúnmente se entiende como sujeto y objeto, o sociedad y naturaleza, el libro en su conjunto produce algo bastante interesante: una serie de textos donde las relaciones humanas nunca son tan humanas, y una materialidad que nunca pierde su impulso político.

Piedra, papel y tijera, en resumen, constituye una contribución interesante a la discusión sobre instrumentos científicos por su énfasis en la ambigüedad y en los múltiples aspectos que discute de los objetos, así como por su insistencia en la interacción entre países que producen y consumen instrumentos científicos, y por el espacio que crea entre enfoques inspirados en el giro material y en el materialismo marxista.

\section{NOTA}

${ }^{1}$ Para la conexión entre el giro material y los instrumentos científicos, ver sobre todo Van Helden y Hankins (1994); Latour y Woolgar (1986, publicado inicialmente en 1979); y Taub (2011). El giro material y los estándares aparecen por ejemplo en Lampland y Star (2009), y Arellano (2011). Su relación con infraestructura es visible en trabajos como los de de Vetter (2008); Larkin (2013); y Harvey y Knox (2015).

\section{REFERENCIAS}

ARELLANO, Antonio.

Representación matemática de una terapéutica: circulación de inscripciones tecnocientíficas en el tratamiento de la litotripsia extracorporal. História, Ciências, Saúde-Manguinhos, v.18, n.3, p.829-849. 2011.
CHÁZARO, Laura; ACHIM, Miruna; VALVERDE, Nuria (Ed.).

Piedra, papel y tijera: instrumentos en las ciencias en México. Ciudad de México: Universidad Autónoma Metropolitana. 2018. 
HARVEY, Penny; KNOX, Hannah.

Roads: an anthropology of infrastructure and expertise. Ithaca: Cornell University Press. 2015.

LAMPLAND, Martha; STAR, Susan Leigh (Ed.). Standards and their stories: how quantifying, classifying and formalizing practices shape everyday life. Ithaca: Cornell University Press. 2009.

LARKIN, Brian.

The politics and poetics of infrastructure. Annual Review of Anthropology, v.42, p.327-343. 2013.

LATOUR, Bruno; WOOLGAR, Steve.

Laboratory life: the construction of scientific facts. Princeton: Princeton University Press. 1986.
TAUB, Liba.

Introduction: reengaging with instruments. Osiris, v.102, n.4, p.689-696. 2011.

VAN HELDEN, Albert; HANKINS, Thomas L. Introduction: Instruments in the history of science. Osiris, v.9, p.1-6. 1994.

VETTER, Jeremy.

Field science in the railroad era: the tools of knowledge empire in the American West, 18691916. História, Ciências, Saúde - Manguinhos, v.15, n.3, p.597-613. 2008. 